\title{
DisCURSO Y CONOCIMIENTO: UNA MIRADA A LOS ESTUDIOS CRíticos Epistémicos del Discurso Entrevista a Teun A. van Dijk
}

\section{Oscar Iván Londoño Zapata*}

Los Estudios Críticos Epistémicos del Discurso (ECED) son una parte de los Estudios Críticos del Discurso; se enfocan de manera especial sobre cómo el conocimiento y el discurso de los miembros de los grupos dominantes pueden abusar del poder, por ejemplo, en formas de manipulación o exclusión.

Teun A. van Dijk

Teun A. van Dijk presentó bajo el título Discourse and Knowledge. A sociocognitive approach (2014) su más reciente investigación sobre discurso y conocimiento. Este trabajo constituye uno de sus cinco proyectos generales: (i) Estudios Críticos del Discurso (ECD), (ii) Racismo y discurso, (iii) Discurso e ideología, (iv) Teoría del contexto y (v) Discurso y conocimiento. El libro, publicado por Cambridge University Press, es un exhaustivo estudio multidisciplinario que indaga las relaciones entre dos nociones centrales no solo para los Estudios del Discurso (ED) y los Estudios Críticos del Discurso (ECD), sino en general para las ciencias sociales y humanas. Aunque el discurso y el conocimiento han sido estudiados por múltiples campos de saber (epistemología, psicología cognitiva y social, sociología, antropología, lingüística, semiótica, entre otros), por primera vez se construye un enfoque que da cuenta de la dinámica simbiosis entre estas dos nociones y de la necesidad de estudiar el conocimiento desde una mirada multidisciplinar e integradora. En esta entrevista ${ }^{1}$ el lingüista holandés da a conocer algunos elementos generales sobre su investigación.

Licenciado en Lengua Castellana y Magíster en Educación de la Facultad de Ciencias de la Educación de la Universidad del Tolima. Miembro de la Asociación Latinoamericana de Estudios del Discurso, ALED. Docente de Tiempo Completo del Departamento de Estudios Interdisciplinarios del Instituto de Educación a Distancia (IDEAD) de la Universidad del Tolima, Colombia.

1 Esta entrevista fue realizada en noviembre de 2013 a través de comunicación electrónica. 
OILZ: ¿En qué radica su interés por el estudio del conocimiento en relación con el discurso?

TAVD: Mi interés por el estudio del conocimiento y el discurso se desarrolló en la década de los años ochenta del siglo pasado, debido a que desde aquella época me interesó el tema de la cognición social, así como las formas en las que esta influye en los discursos. Después de mis trabajos en aquellos años sobre prejuicios racistas en el discurso (van Dijk, 2010), me enfoqué -en la siguiente década- en la ideología como cognición social. Fruto de estas indagaciones es mi libro Ideología. Una aproximación multidisciplinaria (1999). Mi propósito fue escribir cuatro libros sobre ideología, uno de ellos enfocado en la relación entre ideología y cognición social. Cuando empecé este nuevo texto, hace ya varios años, me di cuenta de que no se puede hablar sobre ideología como cognición social si no se trata en primer lugar -y más fundamentalmente- el conocimiento. De hecho, en mi libro sobre la ideología concluí que esta presupone -y se basa en- conocimientos. Por tanto, antes de continuar con la serie de trabajos sobre este tema decidí construir un texto acerca del discurso y su relación con el conocimiento. Pero ese proyecto editorial se atrasó varios años debido a la investigación sobre el contexto, otra de mis nociones principales en Estudios Críticos del Discurso.

OILZ: Cuéntenos acerca de su libro Discourse and Knowledge. A sociocognitive approach.

TAVD: Al igual que mis últimas publicaciones sobre el contexto (van Dijk, 2011; 2012), el libro Discourse and Knowledge. A sociocognitive approach es un estudio multidisciplinar sobre las relaciones entre discurso y conocimiento. La idea central de dicha relación es que para producir o comprender el discurso se necesita gran cantidad de conocimiento, y mucho de este se aprende a través del discurso.

El libro se estructura a través de varios apartados. En el capítulo inicial, Elements of a Theory of Natural Knowledge, abordo la concepción filosófica del conocimiento en la epistemología, pero desde su definición como conocimiento natural que usa la gente, y no a partir de una concepción abstracta y formal, como se significa en filosofía. Posteriormente, incluyo un apartado desde la psicología cognitiva acerca de las representaciones y los procesos mentales del uso del conocimiento en la producción y comprensión del discurso: Discourse, Knowledge and Cognition. 
El capítulo sobre cognición social, Discourse, Knowledge and Social Cognition, aborda el conocimiento y sus relaciones con el discurso, en términos de representaciones sociales compartidas por comunidades epistémicas y su reproducción en la sociedad. La sociología del conocimiento trata las instituciones epistémicas como los medios de comunicación y las universidades -que a la vez son instituciones discursivas-. Lo anterior se amplía en Discourse, Knowledge and Society. El capítulo más antropológico, Discourse, Knowledge and Culture, examina la relación entre conocimiento y cultura, e indaga acerca de cómo las comunidades epistémicas en el mundo usan diferentes criterios de verdad. Finalmente, en el extenso capítulo sobre lenguaje, discurso y conocimiento (Language, Discourse and Knowledge) reflexiono sobre nociones conocidas como tópico, comentario, foco, modalidad, evidencialidad, presuposiciones, entre otras, pero desde una perspectiva más discursiva que gramatical.

OILZ: Al plantear que el conocimiento es un saber natural que usa la gente en diversos contextos, se involucran otros conocimientos (cotidianos, populares, del sentido común) que no son considerados por la epistemología hegemónica como conocimientos científicos (verdaderos, confiables) sino como creencias o simples ideas. ¿Cuál es su posición al respecto? ¿Qué implicaciones ideológicas podría tener lo anterior?

TAVD: En mi libro abordo el conocimiento en general y no el conocimiento científico. Para el conocimiento natural, cotidiano, popular, también se usan criterios epistémicos como la experiencia (Yo lo vi con mis propios ojos...), el discurso (Lo he leído en el periódico..., ¡Me lo dijo Pedro...!) o la inferencia (Él se encontraba en casa porque su coche estaba en frente de esta...). Lo anterior no quiere decir que el conocimiento cotidiano sea necesariamente verdadero sino que cada comunidad epistémica, tanto la científica (que involucra las ciencias sociales, humanas y naturales, y sus diferencias) como la comunidad epistémica de la vida cotidiana, usan sus propios criterios de verdad; aunque estos son, en general, más estrictos en las ciencias; pero los principios son los mismos.

OILZ: ¿Se podría plantear que Discourse and Knowledge. A sociocognitive approach es la primera monografía que estudia sistemáticamente las relaciones entre discurso y conocimiento? 
TAVD: Existe una gran cantidad de documentos en variadas disciplinas sobre conocimiento y muchos otros acerca del discurso, pero creo que mi libro es el primer trabajo que articula las dos nociones en una monografía, y de una manera multidisciplinar. Sin embargo, hay muchos textos que abordan algunos aspectos lingüísticos, por ejemplo, en relación con el tópico, el foco, la modalidad o la evidencialidad.

OILZ: ¿Podría ampliar sus planteamientos acerca de las relaciones entre conocimiento y discurso?

TAVD: Las relaciones básicas entre conocimiento y discurso consisten, por un lado, en el hecho de que la producción y la comprensión del discurso son imposibles sin conocimiento compartido -sin Common Groundentre participantes de la comunicación. De igual manera, mucho de este se adquiere precisamente a través del discurso, durante la socialización epistémica de los miembros de una comunidad. Además, el discurso expresa o señala de muchas maneras este conocimiento compartido o sus fuentes, por ejemplo: a través de presuposiciones, evidencialidad, tópico, foco, artículos definidos, entre otros.

OILZ: ¿Qué papel cumple el discurso en la reproducción social del conocimiento?

TAVD: El conocimiento social se adquiere a través de tres maneras fundamentales: por las experiencias (observación), el discurso y las inferencias de conocimientos que ya existen. Por tanto, el discurso constituye una fuente central en la adquisición de todos los conocimientos, fuera de las experiencias cotidianas directas realizadas por medio de los sentidos (visión, audición, tacto, entre otros). Así, el conocimiento compartido de una comunidad epistémica en general se reproduce a través de los discursos, por ejemplo, a través de la conversación, los medios de comunicación, los libros de texto, el internet y todos los discursos públicos en general.

OILZ: ¿De qué manera se define el conocimiento en el marco de sus investigaciones en Estudios del Discurso?

TAVD: Tradicionalmente, el conocimiento se define, desde la epistemología, como el conjunto de creencias verdaderas justificadas (Justified true beliefs). Nuestra perspectiva más 'naturalista' y pragmática del conocimiento no lo define en términos de la verdad absoluta, sino desde la idea de lo que se toma por verdad en una comunidad 
epistémica. De tal forma, lo que para las personas de la Edad Media era (se presuponía) conocimiento verdadero: la tierra es plana, o-más tardeque el sol gira alrededor de la tierra, ahora se define como una creencia falsa. Esto quiere decir que lo relevante no es lo que es falso o verdadero sino lo que funciona como conocimiento en una comunidad, y según los criterios de la comunidad: observación, discurso o inferencia confiable.

OILZ: Aquello que "funciona como conocimiento en una comunidad" puede contribuir al abuso de poder y al control. Dr. van Dijk, ¿cómo evitar que esto que se toma como "verdad" por una comunidad epistémica (re)produzca la dominación?

TAVD: Cualquier recurso limitado de una comunidad se puede usar para dominar y discriminar. Esto es tan cierto para el dinero, como para la fama o el conocimiento. Toda la educación se basa sobre la construcción de conocimientos compartidos en una sociedad, pero no todas las personas tienen acceso a la misma educación $\mathrm{u}$ otras fuentes de conocimiento. De esta manera, en cada sociedad hay diferencias de conocimiento $y$, en consecuencia, de poder, por lo que hay especialistas en todas las áreas, no solamente académicas. En sí, esas diferencias no son negativas. Lo que es negativo es al abuso de poder de las personas o grupos especializados, porque el abuso de poder epistémico se puede manifestar en formas de manipulación: hacer cosas a las personas de manera que estas no redundan en sus propios intereses sino en los de la persona o grupo dominante. Lo anterior acontece con profesores, médicos, periodistas y políticos entre muchos otros grupos. Resistir contra esto consiste en pedir ayuda a otros especialistas, quienes pueden analizar críticamente esas formas de abuso; de igual forma, contribuir de manera activa en la formación crítica de consumidores, pacientes, estudiantes, entre otros, resulta una acción de resistencia a tal control y dominación.

OILZ: ¿Por qué el estudio del conocimiento debe ser uno de los objetivos fundamentales de las ciencias sociales y humanas?

TAVD: El conocimiento es la base no solamente del uso de la lengua, el discurso y la comunicación sino de todos los actos, interacciones o prácticas sociales. No hay interacción -ni sociedad humana- sin conocimiento compartido.

OILZ: ¿Qué criticas establece a las formas como se ha estudiado el conocimiento en las ciencias sociales y humanas? 
TAVD: En primer lugar, en mi trabajo presento una crítica a la epistemología tradicional que propone una definición formal y no natural del conocimiento, entendido este como las creencias compartidas basadas sobre criterios epistémicos de una comunidad. De esta forma, la definición del conocimiento en epistemología no da cuenta de su naturaleza y función en la sociedad, la interacción y el discurso; al contrario, conforma un significado abstracto, de 'verdad' absoluta. En psicología cognitiva, por su parte, existen trabajos sobre la organización mental del conocimiento, por ejemplo, de estructuras conceptuales, prototipos, guiones (scripts) y, en la actualidad, acerca de estructuras multimodales del cerebro basadas en las experiencias; no obstante, estas indagaciones ignoran la dimensión psicosocial del conocimiento como estructura sociocognitiva compartida por las comunidades. Lo anterior se da excepto en la psicología del discurso. La filosofía y las ciencias sociales abordan el conocimiento sin tratar sus dimensiones cognitivas y discursivas. Es por estas razones que mi libro se configura a través de una mirada multidisciplinar.

OILZ: ¿Y específicamente en los Estudios Críticos del Discurso (ECD)?

TAVD: Debido a que todo estudio del discurso necesita una base epistémica, también los Estudios Críticos del Discurso la tienen. Además, los ECD estudian específicamente el abuso del poder que se da a través del discurso. Por tanto, después de los planteamientos de Michel Foucault, los ECD puede examinar cómo el discurso puede ser dominante cuando manipula el conocimiento de la personas, excluye oyentes o lectores al presuponer conocimientos que ellos no tienen, entre otros aspectos.

OILZ: ¿Qué papel cumple el conocimiento en la relación discurso, cognición y sociedad?

TAVD: Desde mi perspectiva, el conocimiento es la base de toda cognición social y, por consiguiente, de todo discurso, interacción y comunicación tanto de la sociedad humana como de sus comunidades epistémicas.

OILZ: ¿Porquéel enfoquesociocognitivo, desde una miradamultidisciplinaria, es capaz de proporcionar una visión integral del conocimiento y el discurso?

TAVD: Como muchas otras nociones (discurso, ideología, poder...), el conocimiento es un fenómeno complejo que necesita una aproximación multidisciplinar. No se puede reducir a la cognición pura, ni a lo social únicamente, de ahí la relevancia de nuestra aproximación sociocognitiva. 


\section{OILZ: ¿Cómo se representa el conocimiento en la mente?}

TAVD: Esto es un problema fundamental de la psicología cognitiva. Hay varias propuestas desde los años sesenta y setenta del siglo pasado: estructuras jerárquicas de conceptos (un coche es un tipo de medio de transporte), organización en conjuntos de prototipos (un pingüino no es un pájaro prototípico), guiones o scripts (el conocimiento que tenemos sobre el evento de ir al cine, con setting espacio-temporal, participantes, actos, objetivos, entre otros) o una representación multimedia basada sobre nuestras experiencias y organizada por diferentes partes del cerebro: lo que sabemos de un coche no es solamente conceptual, sino también visual, auditivo, la experiencia "motor" de conducir, la emoción (el placer de conducir, o el miedo al tráfico). Para mí esa concepción multimodal del conocimiento es más bien una característica de los modelos mentales individuales, que representan la experiencia individual en la Memoria Episódica. El conocimiento sociocultural es compartido $y$, por tanto, muchas veces se abstrae de la experiencia individual. Un conductor tiene experiencias diferentes sobre su coche en comparación con las de un peatón, pero los dos tienen el mismo concepto de coche, que es sociocultural. Por eso pueden comunicarse entre ellos, o comprender un artículo en el periódico sobre un accidente de coche.

OILZ: ¿De qué manera se articula esta teoría del conocimiento y del discurso con sus nuevos planteamientos sobre el contexto?

TAVD: En mis trabajos sobre esta noción afirmo que los contextos son modelos subjetivos de los participantes sobre las dimensiones fundamentales de la situación de comunicación. Una de esas dimensiones es el conocimiento: lo que los hablantes saben acerca del conocimiento de los oyentes, el Common Ground. Sin ese conocimiento no se puede hablar (o escribir) de manera adecuada. En cada oración los hablantes muestran lo que saben sobre los conocimientos de los oyentes, como por ejemplo en el uso de artículos definidos o presuposiciones.

OILZ: ¿Qué aportes realizan sus planteamientos sobre discurso y conocimiento a la teoría de los modelos mentales?

TAVD: Los modelos mentales son representaciones personales y contextuales de los participantes sobre eventos, actos o situaciones concretos. Estos modelos pueden ser formas de conocimientos individuales, pero también se basan sobre conocimientos socioculturales 
genéricos. Por otro lado, los conocimientos genéricos se pueden construir desde los modelos específicos, por ejemplo, por generalización, abstracción y descontextualización. Lo que sabemos sobre el terrorismo se construyó sobre la base de muchos textos en los medios de comunicación que expresan modelos mentales acerca de eventos concretos del terrorismo.

OILZ: ¿Por qué el conocimiento debe ser analizado como un tipo de creencia social compartida?

TAVD: Sin una definición del conocimiento como creencia compartida no se pueden describir ni explicar la interacción humana, la comunicación y el discurso.

OILZ: ¿Qué implicaciones tiene el conocimiento y el discurso en las ideologías?

TAVD: Las ideologías también son formas de cognición social, son creencias compartidas, pero no por toda una comunidad epistémica, sino por grupos ideológicos dentro de esta. De tal forma, las ideologías presuponen conocimientos.

OILZ: ¿Qué es el dispositivo K?

TAVD: El dispositivo $K$ (dispositivo del conocimiento) es un constructo teórico de los modelos contextuales. Representa la manera en la que los participantes del discurso manejan el Common Ground, es decir, el conocimiento compartido. El dispositivo controla todas las expresiones y presuposiciones de conocimiento del discurso y define su adecuación epistémica.

OILZ: ¿Qué estructuras discursivas pueden ser controladas por el dispositivo $K$ ?

TAVD: Casi todas. Desde los artículos definidos, el orden de palabras (tópico y foco), las presuposiciones, los derechos y las responsabilidades en la conversación, hasta las expresiones evidenciales, modalidades, definiciones, entre otros.

OILZ: ¿Qué es la evidencialidad? ¿Qué papel cumple en su teoría sobre el conocimiento?

TAVD: La evidencialidad es la manera en la que el discurso indica de qué fuente obtiene el hablante su conocimiento. En algunas lenguas, en 
general indígenas, esto se expresa incluso en la morfología del verbo, si el hablante sabe algo por la visión, el oído, el tacto o el discurso.

OILZ: ¿Son los modelos mentales y los contextuales la interfaz entre el conocimiento y el discurso?

TAVD: No solamente son la interfaz entre discurso y conocimiento sino, más generalmente, entre situación de comunicación y discurso. El conocimiento es solo una dimensión de la situación de comunicación, puesto que también están presentes la identidad, los roles y las relaciones entre participantes y sus objetivos, o el tiempo y el lugar de la comunicación.

OILZ: ¿Cuáles serían los principales fundamentos de los Estudios Críticos Epistémicos del Discurso (ECED?

TAVD: Los Estudios Críticos Epistémicos del Discurso (ECED) son una parte de los Estudios Críticos del Discurso: se enfocan de manera especial sobre cómo el conocimiento y el discurso de los miembros de los grupos dominantes pueden abusar del poder, por ejemplo, en formas de manipulación o exclusión.

OILZ: Sus investigaciones han evidenciado distintos modos de gestionar y manipular el conocimiento en diversos niveles de organización del discurso. ¿Cómo se manipula el conocimiento para ejercer control sobre el discurso?

TAVD: Debido a que el conocimiento compartido (Common Ground) es fundamental para cada discurso adecuado, se puede manipular a los oyentes o a los lectores de muchas maneras: contar lo que ya saben, presuponer lo que no pueden saber, expresar como conocido lo que no lo es, entre otras estrategias.

OILZ: ¿Ha trabajado usted en una tipología del conocimiento?

TAVD: He trabajado sobre la tipología del conocimiento en algunos artículos, pero estos planteamientos son provisionales y, como cualquier tipología, presupone una teoría sistemática de base. Así, se pueden distinguir, teóricamente, los conocimientos por sus objetos de conocimiento (personas, grupos, animales, objetos culturales, físicos, entre otros), que tienen una semántica y una gramática diferentes en diversas lenguas; igualmente, por la comunidad y los criterios epistémicos (conocimiento científico, popular, entre otros); por la certeza del conocimiento (como se expresa a través de las modalidades); por la fuente del conocimiento 
(experiencia, discurso o inferencia) como se expresa en la evidencialidad; por abstracto vs. concreto, entre otros. Sin duda, estas tipologías se pueden elaborar más.

OILZ: ¿Quéaportes realiza esta nueva teoría del discurso y del conocimiento a los procesos de enseñanza y aprendizaje?

TAvD: Todavía no he reflexionado en profundidad acerca del uso de esta teoría en la educación, pero sin duda hay aplicaciones en la estructuras de los libros de texto, en la enseñanza en el aula, en los artículos de divulgación en la prensa y en programas de televisión, entre otros. El aprendizaje es adquisición de conocimiento, y mucho de este conocimiento se aprende por el discurso y, por tanto, comprender la relación entre discurso y conocimiento es crucial para todas estas áreas de aplicaciones.

OILZ: Finalmente, ¿sobre qué otra noción o tema trabajará de ahora en adelante? Cuéntenos acerca de sus proyectos futuros.

TAVD: Mi libro sobre discurso y conocimiento fue concebido como parte preliminar de un libro de ideología y cognición, que sería una continuación de mi trabajo Ideología. Una aproximación multidisciplinaria (1999). Es posible que continúe con la serie de libros sobre ideología; en general me fascina la relación entre discurso y cognición social, sobre todo desde una perspectiva crítica y social. ¡Ya veremos! 


\section{Referencias}

Van Dijk, Teun A. (1999). Ideología. Una aproximación multidisciplinaria. Barcelona, España, Editorial Gedisa.

Van Dijk, Teun A. (2010). Prejuicio en el discurso. Análisis del prejuicio étnico en la cognición y en la conversación. Sevilla, España, ArCiBel Editores.

Van Dijk, Teun A. (2011). Sociedad y discurso. Cómo influyen los contextos sociales sobre el texto y la conversación. Barcelona, España, Editorial Gedisa.

Van Dijk, Teun A. (2012). Discurso y contexto. Un enfoque sociocognitivo. Barcelona, España, Editorial Gedisa.

Van Dijk, Teun A. (2014). Discourse and Knowledge. A sociocognitive approach. Londres, Inglaterra, Cambridge University Press. 